\title{
Imprints of independent allopolyploid formations on patterns of gene expression in two sibling yarrow species (Achillea, Asteraceae)
}

Duo Chen ${ }^{1 \dagger}$, Peng-Cheng Yan $^{2+}$ and Yan-Ping Guo ${ }^{1 *}$ (i)

\begin{abstract}
Background: Polyploid species often originate recurrently. While this is well known, there is little information on the extent to which distinct allotetraploid species formed from the same parent species differ in gene expression. The tetraploid yarrow species Achillea alpina and A. wilsoniana arose independently from allopolyploidization between diploid A. acuminata and A. asiatica. The genetics and geography of these origins are clear from previous studies, providing a solid basis for comparing gene expression patterns of sibling allopolyploid species that arose independently.
\end{abstract}

Results: We conducted comparative RNA-sequencing analyses on the two Achillea tetraploid species and their diploid progenitors to evaluate: 1) species-specific gene expression and coexpression across the four species; 2) patterns of inheritance of parental gene expression; 3) parental contributions to gene expression in the allotetraploid species, and homeolog expression bias. Diploid A. asiatica showed a higher contribution than diploid A. acuminata to the transcriptomes of both tetraploids and also greater homeolog bias in these transcriptomes, possibly reflecting a maternal effect. Comparing expressed genes in the two allotetraploids, we found expression of ca. 30\% genes were species-specific in each, which were most enriched for GO terms pertaining to "defense response". Despite species-specific and differentially expressed genes between the two allotetraploids, they display similar transcriptome changes in comparison to their diploid progenitors.

Conclusion: Two independently originated Achillea allotetraploid species exhibited difference in gene expression, some of which must be related to differential adaptation during their post-speciation evolution. On the other hand, they showed similar expression profiles when compared to their progenitors. This similarity might be expected when pairs of merged diploid genomes in tetraploids are similar, as is the case in these two particular allotetraploids.

Keywords: Allopolyploid speciation, RNA-sequencing, Inheritance of gene expression, Homeolog express bias, Achillea

\footnotetext{
* Correspondence: guoyanping@bnu.edu.cn

${ }^{\dagger}$ Duo Chen and Peng-Cheng Yan contributed equally to this work.

'Key Laboratory of Biodiversity Science and Ecological Engineering of the

Ministry of Education, and College of Life Sciences, Beijing Normal University, Beijing, China

Full list of author information is available at the end of the article
}

C C The Author(s). 2021 Open Access This article is licensed under a Creative Commons Attribution 4.0 International License, which permits use, sharing, adaptation, distribution and reproduction in any medium or format, as long as you give appropriate credit to the original author(s) and the source, provide a link to the Creative Commons licence, and indicate if changes were made. The images or other third party material in this article are included in the article's Creative Commons licence, unless indicated otherwise in a credit line to the material. If material is not included in the article's Creative Commons licence and your intended use is not permitted by statutory regulation or exceeds the permitted use, you will need to obtain permission directly from the copyright holder. To view a copy of this licence, visit http://creativecommons.org/licenses/by/4.0/. The Creative Commons Public Domain Dedication waiver (http://creativecommons.org/publicdomain/zero/1.0/) applies to the data made available in this article, unless otherwise stated in a credit line to the data. 


\section{Background}

Polyploidy is an important mechanism of plant speciation. In an allopolyploid species, the combined effects of two or more diverged subgenomes and their regulatory interactions can lead to a myriad of genetic and epigenetic modifications described as genomic and transcriptomic shock [1-6]. The resulting changes in gene expression may often generate phenotypic variation affecting individual fitness and evolution of allopolyploids [7-13]. Analyses of synthetic polyploid plants have demonstrated that genomic and transcriptomic shock usually occurs immediately after polyploidization [1, 1417], though changes may also take place during later stages of the evolutionary history of a polyploid species [3, 6, 18, 19].

Polyploid species often consist of lineages that originated independently and recurrently from the same parental species [20, 21]. Such recurrent formation can result in karyotypic, genomic, transcriptomic and phenotypic variation across lineages as demonstrated in recently originated allotetraploid species of Tragopogon (Asteraceae) [22-27]. However, whereas different lineages of the same allopolyploid species have been studied in detail, divergent species derived by independent origins from the same parental species have been reported less frequently and studied less [28-30]. Only in the orchid genus, Dactylorhiza, has research been conducted on gene expression and epigenetic differences among sibling allotetraploids derived from the same parental species pair. This showed that both kinds of differences occurred and were stable among these allotetraploid species, raising the possibility that they reflect divergent adaptation to the different environmental conditions experienced by the species $[28,29]$.

To shed further light on how gene expression might differ between allopolyploid species that originated independently from the same progenitor species, we focus here on two allotetraploid yarrow species, Achillea alpina L. and A. wilsoniana Heimerl ex Hand. -Mazz., and their parental species, $A$. acuminata (Ledeb.) Sch. -Bip. and $A$. asiatica Serg. (Asteraceae). In China, these tetraploid species have different distributions, with A. alpina occurring in the northeast and $A$. wilsoniana in the southwest of the country [30, 31]. Our previous research indicated that the two tetraploids originated independently 35-80 kya following hybridization between their diploid parents during the megainterstadial before the Last Glacial Maximum. Two independent contacts between the parental species were involved, possibly in deglaciated habitats located near refugia present in the mountains of northeast China and relatively southwestern in the Qinling Mountains, respectively [30]. According to plastid sequencing data, A. asiatica mostly likely acted as the maternal parent of both tetraploids [32, 33].
To investigate transcriptome changes occurred during allopolyploidization and the following long-term evolution, it is not only necessary to check specific and coexpressed genes among progeny and progenitor species, but also to examine total and relative expression levels of homeologous genes in allotetraploids. Relative expression levels of homeologs may reflect preexisting parental relative levels (parental legacy) or originate following allopolyploidy with one homeolog preferentially expressed relative to the other (expression bias) [34-37]. Achillea alpina and A. wilsoniana are ideal for such analysis for the following reasons. First, their parental-offspring relationships are clear and simple (no complicated reticulate relationships are involved according to previous studies). Second, the parental species are extant, making it feasible to compare data from allopolyploids with that of their progenitors. Third, the progenitor species, $A$. acuminata and $A$. asiatica, show high levels of genomic sequence divergence $[32,38]$, while each allopolyploid species maintains both parental genomes intact, having experienced only low levels of homeologous recombination [30, 32, 33]. For these reasons, it is easy to distinguish homeologous genes from each other in the allopolyploid transcriptome, and to measure parental contributions and homeolog expression bias.

In this study, we screened the transcriptome profiles of the two Achillea allotetraploid species and their diploid progenitor species by means of whole transcriptome sequencing. By a comparative analysis of these transcriptomes, we examined first the inheritance of parental gene expression, and second relative parental contributions and homeolog expression bias. From our results, we ask whether parental effects which are frequently found in plant hybrid/allopolyploid transcriptomes, are apparent in the present polyploid system. Furthermore and most importantly, we question to what extent inherited patterns of gene expression are similar in different allopolyploids derived from the same parental species, and how significant evolutionary factors, e.g. natural selection and/or genetic drift, have influenced divergent gene expression profiles of the two independently evolved tetraploid species.

\section{Results}

\section{Transcriptome profiles}

Approximately 34-49 million $100 \mathrm{bp}$ paired-end raw reads were generated for a library of each of the studied Achillea species. After removing adapter sequences and filtering out reads with low quality, 93.2-96.4\% of clean reads were obtained (Table $\mathrm{S} 1$ ). The initial transcripts were assembled and filtered to 51,414-88,150 unigenes across the studied species, with the N50 length of unigenes always longer than the average length of unigenes in each sample (Table 1). The proportion of unigenes with complete or partial ORFs was $63-71 \%$. These unigenes were used for subsequent gene expression analysis 
Table 1 Information of unigenes in the present RNA-Seq data

\begin{tabular}{|c|c|c|c|c|c|}
\hline & acuARX & acuQL & asi & alp & wil \\
\hline Number of assembled transcripts by Trinity & 177,816 & 180,194 & 272,030 & 282,619 & 300,158 \\
\hline Number of unigenes & 51,414 & 55,391 & 59,600 & 81,143 & 88,150 \\
\hline Average length of unigenes (bp) & 1230.20 & 1243.32 & 1074.86 & 976.40 & 1011.16 \\
\hline N50 length of unigenes (bp) & 1678 & 1687 & 1544 & 1386 & 1432 \\
\hline Number of IncRNAs & 5794 & 5794 & 7604 & 11,409 & 13,748 \\
\hline Number of unigenes with no ORF & 9229 & 11,094 & 10,342 & 15,078 & 18,603 \\
\hline Number of unigenes with complete ORF & 21,801 & 24,123 & 20,997 & 24,412 & 27,984 \\
\hline Number of unigenes with partial ORF & 14,590 & 14,380 & 20,657 & 30,244 & 27,815 \\
\hline
\end{tabular}

Abbreviation of accession names: acuARX for Arxan population of A. acuminata; acuQL for Qinling population of $A$. acuminata; alp for A. alpina; asi for A. asiatica; wil for A. wilsoniana

(Table 1). The FPKM values of unigenes showed that data correlation among biological replicates of the same tissue/organ of a species/population was higher than among different tissues/organs, indicating that experimental sampling was repeatable and reliable (Fig. S1).

\section{Specifically expressed and coexpressed genes among} each allotetraploid species and its diploid progenitors As shown in the Venn diagrams (Fig. 1), there were 23, $614(29.1 \%)$ and $27,535(31.2 \%)$ genes showing speciesspecific expression in the allotetraploids A. alpina and A. wilsoniana, respectively, equating to higher proportions than in the diploid parental species (20-25\%) and indicating rather high amounts of novel gene expression in both allotetraploids. The numbers of genes expressed in both parents, but not detected in the allotetraploid transcriptome, were $2150 / 2137$ and 2320/2217 in $A$. alpina and $A$. wilsoniana, respectively, suggesting a relatively low level of gene silencing or loss. With regard to coexpression of genes, 35,286 unigenes (about 43.5\% of all unigenes) were coexpressed between $A$. alpina and both diploid species, and 36,385 (about $41.3 \%$ of all the unigenes) were coexpressed by $A$. wilsoninana and the two diploids (Fig. 1).

Particularly interesting are the genes of each tetraploid specifically coexpressed with each parental species as this indicates the relative contribution of each parent to the transcriptome of each tetraploid. We found that A. alpina specifically coexpressed 9922 unigenes with diploids $A$. acuminata, and 12,321
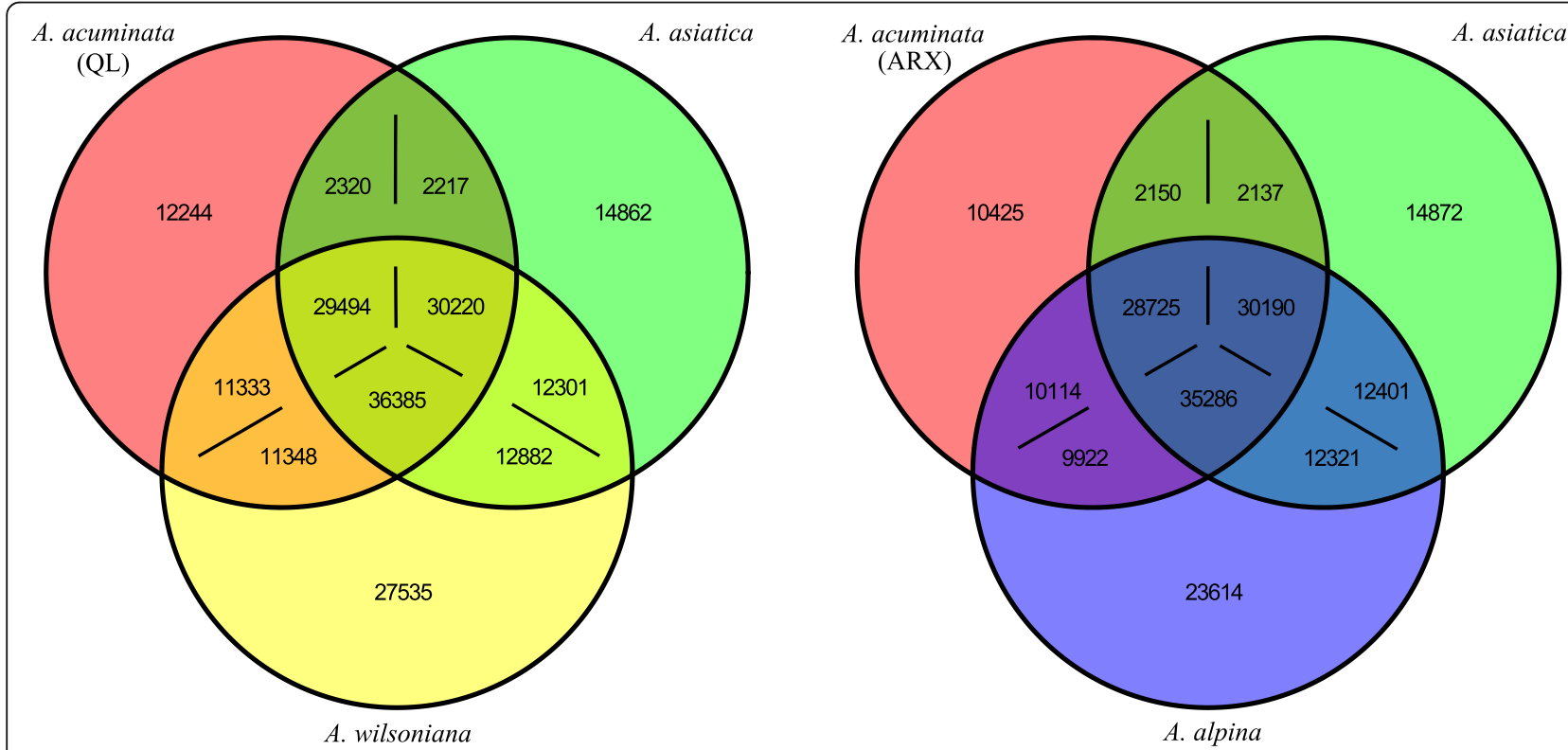

Fig. 1 Venn diagrams showing amounts of coexpressed and specifically expressed genes of the studied allotetraploid species and their diploid progenitors. As the two allopolyploid species originated independently in different regions, and as the diploid A. acuminata shows population genetic differentiation, the analysis was conducted separately for each tetraploid species. In the coexpressed gene category, gene number in each species is given (copy-number on some loci may be different among species). Abbreviations: ARX, Arxan Mt:; QL, Qinling Mts 
unigenes with $A$. asiatica; while $A$. wilsoniana coexpressed 11,348 and 12,882 unigenes with A. acuminata and $A$. asiatica, respectively (Fig. 1). Thus, both tetraploids coexpressed more genes with $A$. asiatica than with $A$. acuminata. Gene Ontology (GO) analysis indicated significant enrichment of these coexpressed genes mostly in terms "response to stress" and "defense response", suggesting that the tetraploid species inherited environmental response genes separately from both progenitors (Fig. 2: A, B; Additional file 6).

\section{Species-specific and coexpressed genes in the two allotetraploid species}

Table 2 shows that comparing the expressed genes in the tetraploids, $29.4 \%$ genes expressed in A. alpina showed species-specific expression and $33.9 \%$ genes expressed in A. wilsoniana were species-specific. Among the coexpressed genes, $78 \%-83 \%$ were expressed equally in both species, while only about $10 \%$ showed up- or down-regulation in one or the other (Table 2). Most enriched GO terms related to biological process (BP) of genes exhibiting species-specific expression pertained to "defense response" in both tetraploids (Fig. 2: C, D; Additional file 7).

In parallel, we found approximately $30 \%$ of genes showing population-specific expression in diploid $A$. acuminata; these were most enriched for GO terms pertaining to "defense response" and/or "response to stress" (Fig. 2: E, F; Additional file 7). Moreover, genes coexpressed by each tetraploid with its sympatric $A$. acuminata population were also most enriched for GO terms related to "response to stress" and "defense response" (Fig. 2: A, B; Additional file 6]). These results imply that

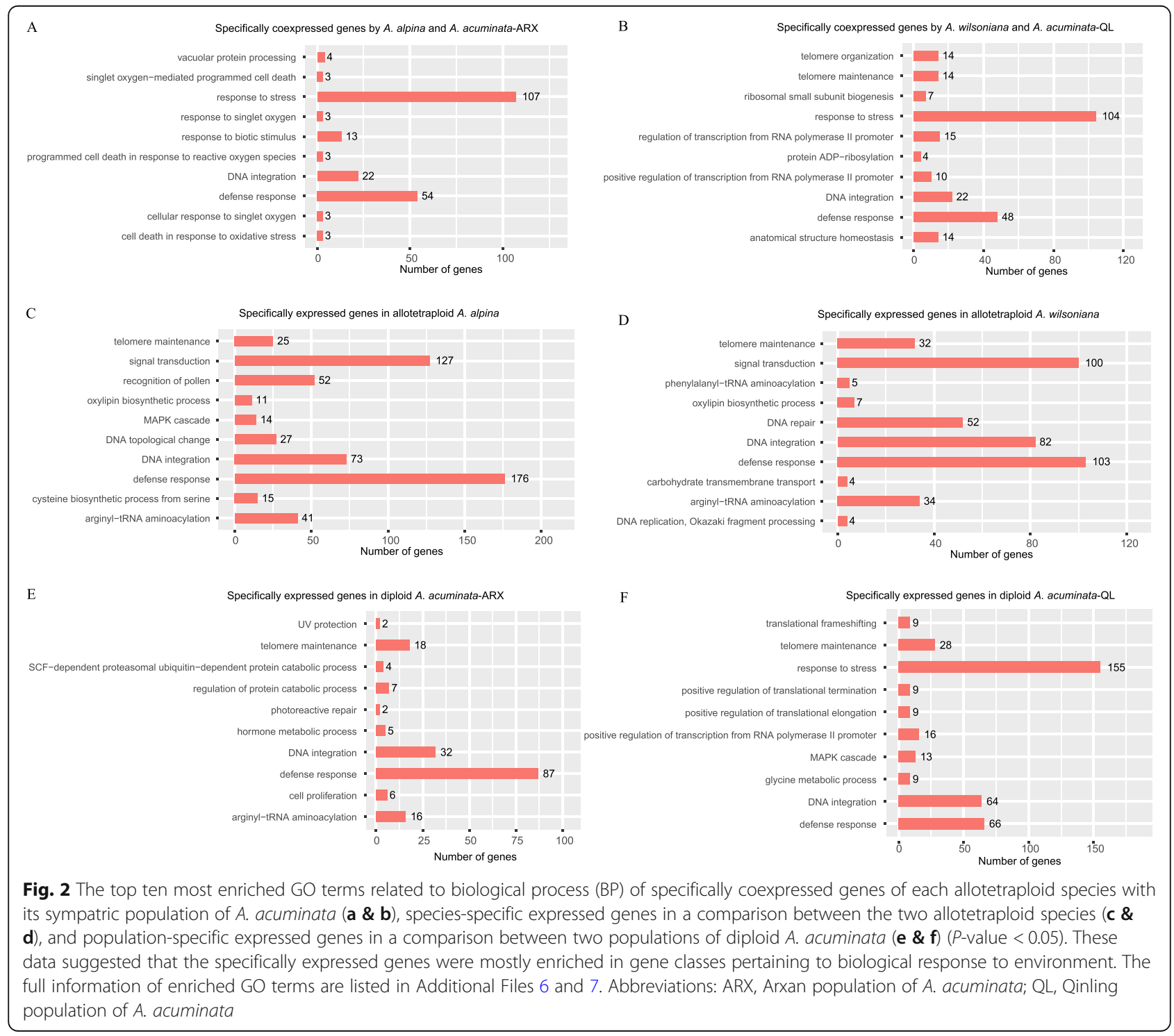


Table 2 Number of specifically and differentially expressed genes in the two studied allotetraploid species

\begin{tabular}{llll}
\hline Specific in A. alpina & Specific in A. wilsoniana & Expressed in both tetraploids (stem apex) & Expressed in both tetraploids (leaf) \\
\hline 23845 (29.4\%) & 29881 (33.9\%) & 4049 (up-regulate in A. alpina) & 2879 (up-regulate in A. alpina) \\
& & 8328 (up-regulate in A. wilsoniana) & 6727 (up-regulate in A. wilsoniana) \\
& 44524 (equal expression in both) & 47267 (equal expression in both)
\end{tabular}

the two geographically separated tetraploids may have inherited genes and expression patterns from their sympatric diploid parental species which could be important in local adaptation.

\section{Inheritance patterns of gene expression}

Figure 3 shows the numbers and proportions of differentially expressed genes (DEGs) among all expressed genes in the allotetraploids. Most of these genes $(71.49 \%$ in $A$. alpina and $67.30 \%$ in A. wilsoniana) were 'conserved', meaning that the total expression of homeologs for a given gene in the allotetraploids was statistically similar to the expression levels of that gene in both parental species.

Altered gene expression in the tetraploids was evidenced by expression inheritance patterns classified into 12 categories. Thus, 5.8 and $5.0 \%$ of expressed genes in A. alpina and A. wilsoniana, respectively, had expression levels intermediate to the parental species (categories I and XII in Fig. 3). Approximately 15\% of genes showed "expression-level dominance" (categories II, XI, IV and
IX) with both tetraploids exhibiting greater $A$. asiatica expression-level dominance (S-dominance) than $\mathrm{A}$. acuminata dominance (C-dominance) (categories IV and IX vs. II and XI). Finally, both tetraploids possessed more transgressively downregulated genes (categories III, VII and $\mathrm{X}$ ) than transgressively upregulated genes (categories V, VI and VIII).

\section{Relative homeolog contribution and homeolog expression bias}

The two allotetraploids displayed a relatively small proportion of silent/lost parental genes. Moreover, they exhibited imbalanced silencing/loss of homeologs between the two parental subgenomes. Silence/loss of genes were more evident for $A$. acuminata-homeologs than for $A$. asiatica-homeologs, implying preferential expression of the $A$. asiatica-subgenome in both tetraploids (Table 3 ).

The relative homeolog contribution to total expression levels of allotetraploid genes was quantified by $\mathrm{Rh}[\mathrm{Rh}=$ $\log _{2} \quad$ (acu-homeolog/asi-homeolog)] (Fig. 4).

\begin{tabular}{|c|c|c|c|c|c|c|c|c|c|c|c|c|c|c|}
\hline \multirow{3}{*}{ Categories } & \multicolumn{2}{|c|}{ Intermediate } & \multicolumn{2}{|c|}{$\begin{array}{l}\text { C-expression } \\
\text { level dominance }\end{array}$} & \multicolumn{2}{|c|}{$\begin{array}{l}\text { S-expression } \\
\text { level dominance }\end{array}$} & \multicolumn{3}{|c|}{$\begin{array}{l}\text { Transgressive down- } \\
\text { regulation }\end{array}$} & \multicolumn{3}{|c|}{$\begin{array}{l}\text { Transgressive up- } \\
\text { regulation }\end{array}$} & \multirow{3}{*}{ Conserved } & \multirow{3}{*}{ Total } \\
\hline & I & XII & II & XI & IV & IX & III & VII & $\mathrm{X}$ & V & VI & VIII & & \\
\hline & $\begin{array}{ll}S & C \\
& \end{array}$ & $\begin{array}{ll}S & C \\
\end{array}$ & $\begin{array}{ll}S & C \\
0\end{array}$ & $\begin{array}{ll}S \quad C \\
D_{0}\end{array}$ & $\begin{array}{ll}S \quad C \\
\bullet\end{array}$ & $\begin{array}{ll}S & C \\
& \end{array}$ & $\begin{array}{ll}S & C \\
& \end{array}$ & $\begin{array}{l}{ }^{\mathrm{C}} \\
V\end{array}$ & $\begin{array}{ll}{ }^{S} & \mathrm{C} \\
\end{array}$ & $\gamma^{\mathrm{S}}$ & 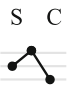 & ${ }^{S C}$ & & \\
\hline $\begin{array}{c}\text { A. alpina } \\
\text { (Stem apex) }\end{array}$ & 1165 & 859 & 1254 & 938 & 1373 & 1568 & 121 & 2569 & 112 & 57 & 33 & 625 & 23962 & 34636 \\
\hline $\begin{array}{l}\text { A. alpina } \\
\text { (Leaf) }\end{array}$ & 1100 & 867 & 1151 & 760 & 1146 & 1389 & 62 & 1988 & 80 & 55 & 17 & 456 & 25558 & 34629 \\
\hline Proportion & \multicolumn{2}{|c|}{$5.76 \%$} & \multicolumn{2}{|c|}{$5.92 \%$} & \multicolumn{2}{|c|}{$7.91 \%$} & \multicolumn{3}{|c|}{$7.12 \%$} & \multicolumn{3}{|c|}{$1.79 \%$} & $71.49 \%$ & ---- \\
\hline \begin{tabular}{|c|} 
A. wilsoniana \\
(Stem apex)
\end{tabular} & 1003 & 758 & 1234 & 1018 & 2063 & 1964 & 150 & 2590 & 184 & 145 & 120 & 1023 & 22270 & 34522 \\
\hline \begin{tabular}{|c|}
$\begin{array}{c}\text { A. wilsoniana } \\
\text { (Leaf) }\end{array}$ \\
\end{tabular} & 972 & 698 & 1149 & 862 & 1639 & 1695 & 110 & 2145 & 104 & 129 & 43 & 780 & 24192 & 34518 \\
\hline Proportion & \multicolumn{2}{|c|}{$4.97 \%$} & \multicolumn{2}{|c|}{$6.17 \%$} & \multicolumn{2}{|c|}{$10.66 \%$} & \multicolumn{3}{|c|}{$7.65 \%$} & \multicolumn{3}{|c|}{$3.24 \%$} & $67.30 \%$ & ---- \\
\hline
\end{tabular}

Fig. 3 Inheritance categories of gene expression of the studied allotetraploid species. The categorization involving 12 states of differential expression (labeled with Roman numeral I-XII) is modified from Rapp et al. (2009) [39]. A cartoon depiction is provided for each of the 12 states, where parental states ( $\mathrm{f}$ for $\mathrm{A}$. asiatica; C for A. acuminata) are on the outer edges and the allotetraploid is in the middle. Dots on the same horizontal line indicate statistically equal expression level, whereas dots on higher or lower horizontal lines refer to significantly higher or lower expression level. The 'Intermediate' states, I and XII, indicate gene expression levels in the allopolyploid being significantly different from, but intermediate between the parental levels. The 'conserved' refers to genes with basically equal expression levels among the allotetraploid and both parental species. The number of genes of each category is given, and the percentage of each category group in all expressed genes is provided 
Approximately two-thirds of homeolog pairs displayed equal expression of parental copies, and the remaining one-third exhibited different expression levels of parental homeologs. Among the differentially expressed homeologous pairs, more exhibited higher expression of the $A$. asiatica copy than the A. acuminata copy.

To determine if the detected differential expression of homeologs is derived from pre-existing differences in parental gene expression levels, or is due to homeolog expression bias, we compared $\mathrm{Rh}$ with the relative expression of orthologs between the parental species, $\mathrm{Rp}$ $\left[\mathrm{Rp}=\log _{2}\right.$ (A. acuminata/A. asiatica) $]$ (Fig. 5). Approximately $79 \%$ of homeolog pairs in the tetraploids displayed vertical inheritance of pre-existing parental expression levels, that is, without expression bias. Among the remaining 21\% homeolog pairs that displayed parental expression bias, S-bias (bias toward $A$. asiatica copy) was more common than $\mathrm{C}$-bias (bias toward $A$. acuminata copy).

To understand the possible influence of expression bias to the relative contribution of the parental homeologs, we integrated data sets of relative homeolog expression level and homeolog bias (Table S2). Of the homeolog pairs showing equal expression of parental copies, 35\% showed expression bias, while the rest simply maintained pre-existing parental expression levels. Of the homeolog pairs with unequal expression levels, most might have resulted from homeolog expression bias. For instance, out of 1396 homeolog pairs showing higher expression level of the A. asiatica copy, 1037 (74.3\%) displayed expression bias toward the A. asiatica copy (Table S2).

\section{Validation of RNA-Seq analysis by RT-qPCR}

To validate the analysis and data obtained by RNAsequencing, differential expression of genes was checked using RT-qPCR assays. Unigenes exhibting different inheritance patterns of gene expression (intermediate expression, A. acuminata/A. asiatica expression-level dominance, transgressive expression) were randomly chosen for RT-qPCR verifying. For all 10 unigenes tested, expression patterns revealed by qRT-PCR assays were consistent with those evident in the RNA-Seq data (Fig. S2), demonstrating the reliability of data produced by RNA-sequencing.

\section{Discussion}

To understand the influence of hybridization and polyploidy on the inheritance of gene expression from parental to allopolyploid species, we conducted a transcriptome analysis on two allotetraploid Achillea species that originated independently from the same two parental species. We evaluated RNA-Sequencing data to determine: $(i)$ species-specific gene expression and coexpression among both tetraploid and progenitor diploid species; (ii) inheritance patterns of parental gene expression; and (iii) parental contribution to gene expression level in the tetraploids, and occurrence of homeolog expression bias.

Gene expression profiles in the allotetraploid species with influence of maternal effect

Both hybridization and polyploidization can alter gene expression between progenitors and allopolyploid offspring by affecting the number of expressed genes and their expression levels. In the present analysis only $3.6 \%-4.7 \%$ (Fig. 1) genes expressed in the diploids were not detected in the tetraploid species, suggesting a low level of gene silencing (or loss). On the other hand, each of the tetraploid species possessed a high proportion (approximately 30\%) of species-specific expressed genes (23,614 out of 81,143 genes in A. alpina and 27,535 out of 88,150 genes in A. wilsoniana, Fig. 1), suggesting that hybridization and polyploidy activate some genes not expressed in the diploids.

In hybrid plants, maternal effects may have a strong influence on morphological, life-history and physiological traits, which can be beneficial if the maternal phenotype is linked to increased fitness [40-43]. The present study showed that global gene expression of both Achillea tetraploids was frequently more similar to $A$. asiatica than to $A$. acuminata, as reflected by the number of coexpressed genes between species, expression-level dominance, relative homeolog contribution, homeolog-specific expression and homeolog expression bias. This similarity to $A$. asiatica suggests a maternal effect on gene expression with both tetraploids previously shown to have had an $A$. asiatica-like ancestor as their maternal parent [32, 33].

It has been suggested that parental expression-level dominance in allopolyploids mainly results from up- or down-regulation of one of the homeologous copies,

Table 3 Number of silent/lost homeologs in the studied allotetraploids

\begin{tabular}{lll}
\hline Samples & Number of silent/lost $\boldsymbol{A}$. asiatica-homeologs (\%) & Number of silent/lost A. acuminata-homeologs (\%) \\
\hline A. alpina (Stem apex) & $362(3.60 \%)$ & $539(5.36 \%)$ \\
A. alpina (Leaf) & $311(3.67 \%)$ & $479(5.65 \%)$ \\
A. wilsoniana (Stem apex) & $370(3.50 \%)$ & $517(4.89 \%)$ \\
A. wilsoniana (Leaf) & $331(3.99 \%)$ & $417(5.02 \%)$ \\
\hline
\end{tabular}




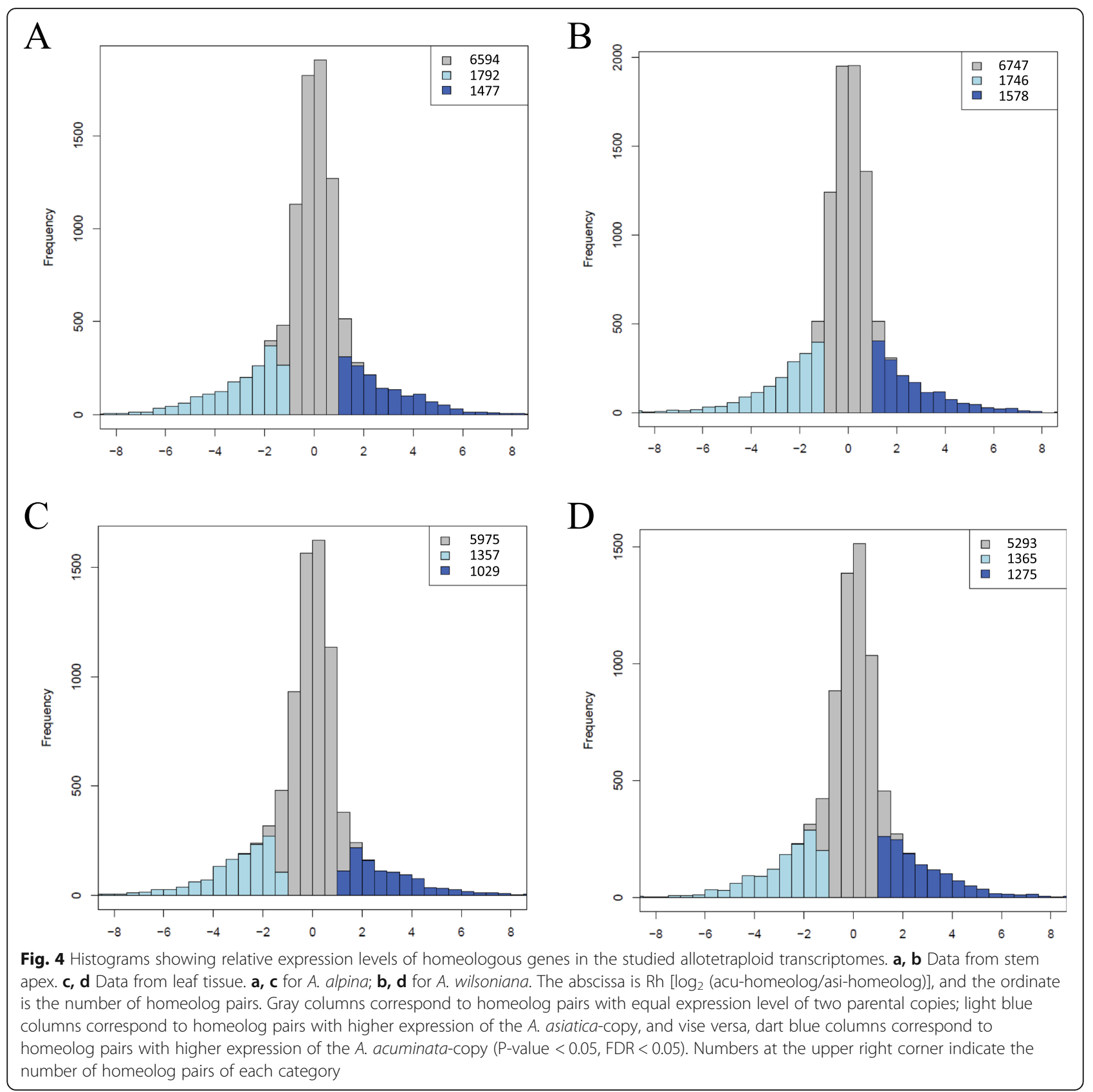

usually of the 'less dominant' parent [44, 45]. Homeolog expression bias may lead to higher expression of one of the parental gene copies due possibly to a difference between parental subgenomes in number and distribution of transposable elements (usually repressing nearby genes), mismatches between parental copies of trans-elements and their target genes, and persistent epigenetic resetting [6, 36, 37, 46, 47]. Maternal effects resulting from one or more of these causes have been reported previously in a number of allopolyploids, e.g. Gossypium hirsutum [18, 48], Spartina anglica [49], Triticum aestivum [50] and Tragopogon miscellus [51].

\section{Comparative global gene expression patterns of allopolyploids independently derived from the same parent species}

Previous research on Dactylorhiza showed that three sibling allotetraploid species derived from the same two parental species were divergent epigenetically and in gene expression, and it was suggested that these 


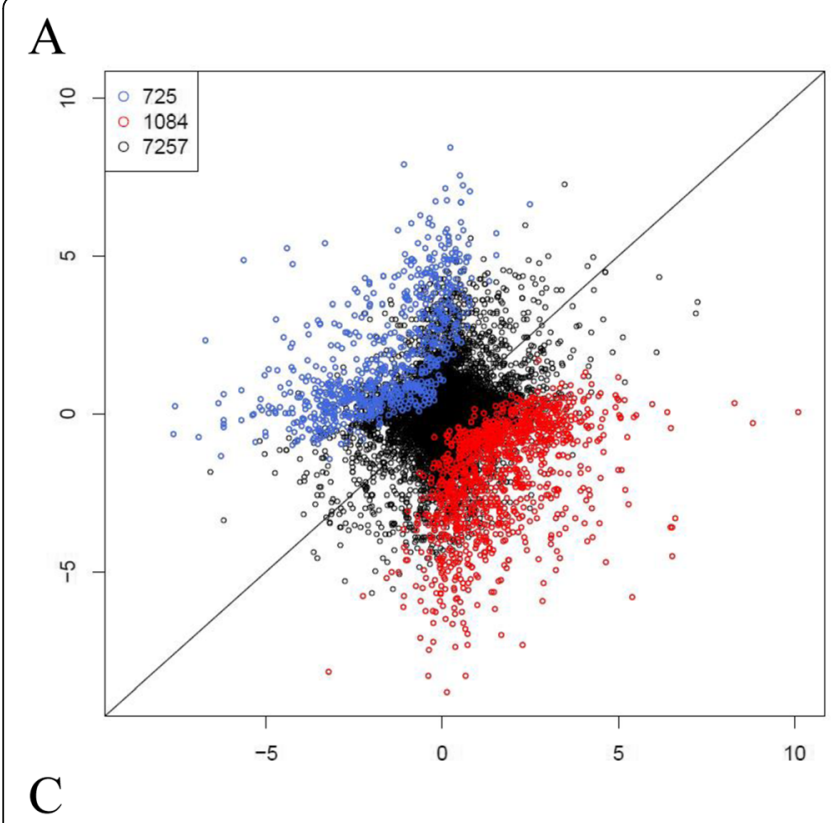

B

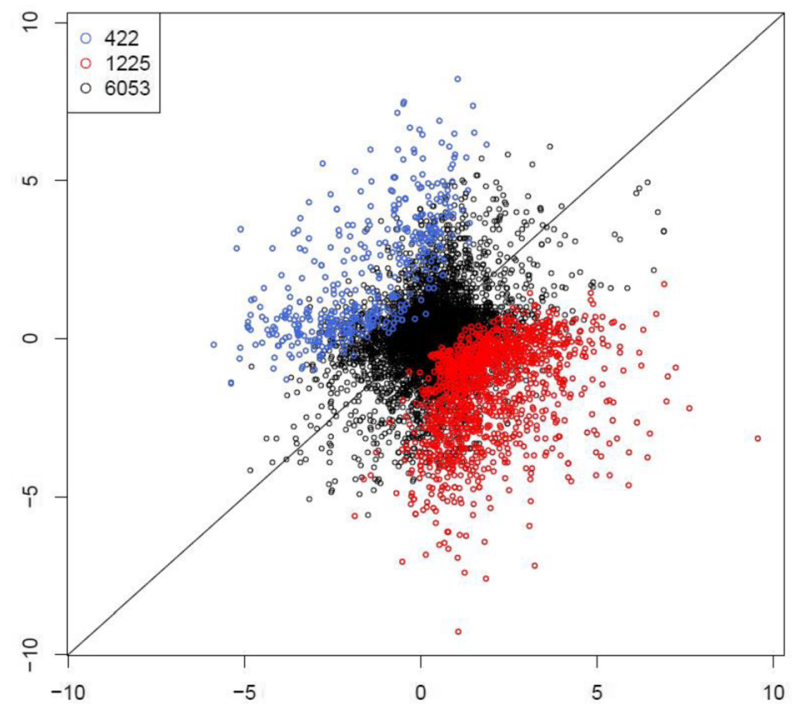

Fig. 5 Scatter plots showing expression bias of homeologs in the studied allotetraploid transcriptomes. $\mathbf{a}, \mathbf{b}$ Data from stem apex. $\mathbf{c}$, $\mathbf{d}$ Data from leaf tissue. a, c for A. alpina. b, $\mathbf{d}$ for A. wilsoniana. The abscissa is Rp [ $\log _{2}$ (A. acuminata/A. asiatica)], and the ordinate is Rh [log 2 (acu-homeolog/ asi-homeolog)]. Black circles correspond to homeolog pairs without expression bias; blue circles correspond to the homeolog pairs with expression bias toward A. acuminata (namely C-bias); and red circles correspond to homeolog pairs with biased expression of the A. asiatica-copy (namely S-bias) (P-value $<0.05, F D R<0.01$, Fisher's exact text). Numbers at the upper left corner indicate the number of homeolog pairs of each category

differences may have been important in their adaptation to different habitats $[28,29]$. Similarly, the two Achillea tetraploids studied here originated independently due to multiple contacts between the same two parental species in different geographical regions, with population genetic analysis showing them to be genetically well-differentiated [30]. Comparing expressed genes in the two tetraploids, we found a high proportion of speciesspecific expression (29.4\% in A. alpina and $33.9 \%$ in $A$. wilsoniana) (Table 2). These species-specific expressed
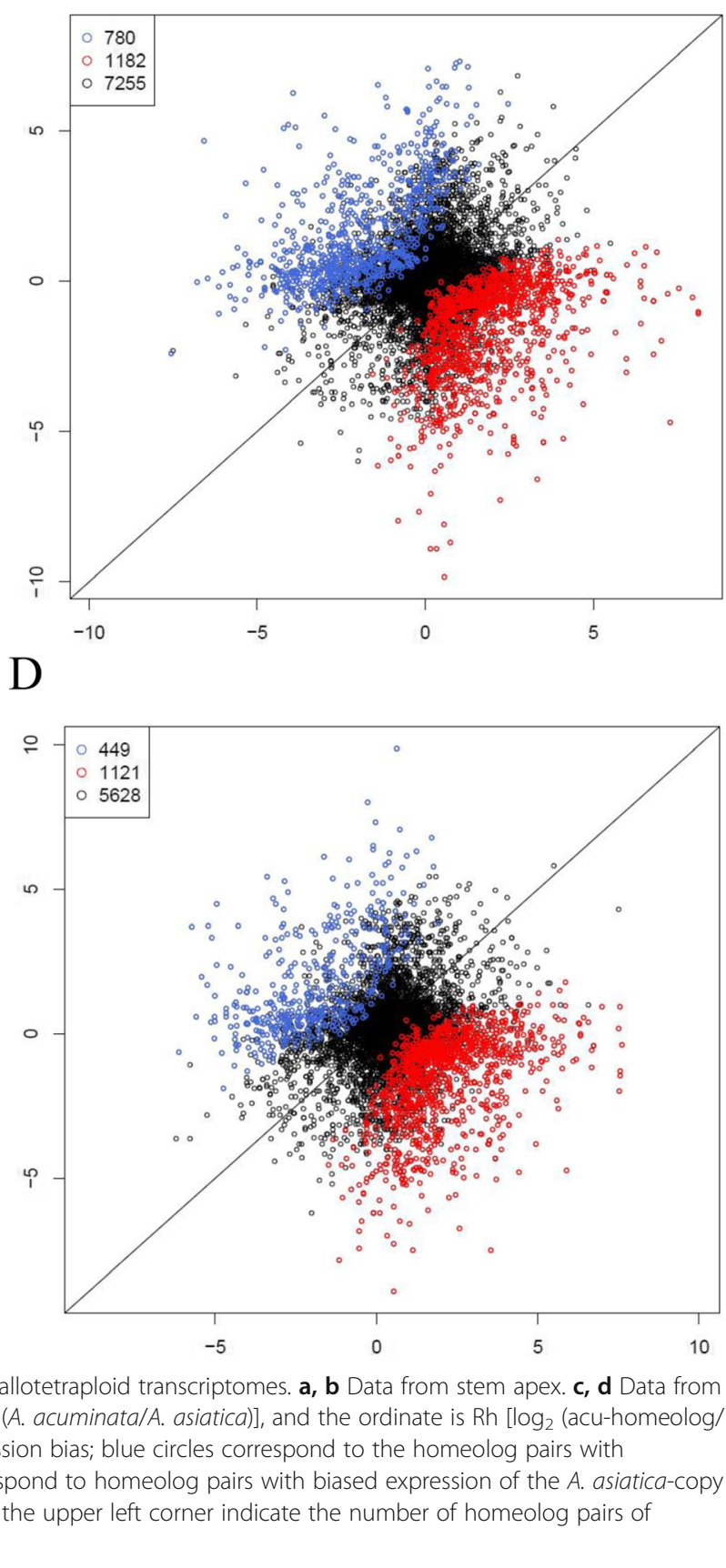

genes were enriched for GO terms pertaining to "defense response" (Fig. 2; Additional file 7). Polyploidy may confer adaptive novelties, as indicated in the aforementioned orchids and in Achillea [9, 13, 28, 29, 39, 52-54]. Speciesspecific and differential expression of genes of $A$. alpina and $A$. wilsoniana might have partly originated as a result of the independent allopolyploidization events that gave rise to these two species, but also to independent postspeciation events due to natural selection and/or genetic drift. 
Despite the species-specific and differentially expressed genes between the two allotetraploids, they display similar transcriptome changes in comparison to their diploid progenitors, e.g., maternal effects of $A$. asiatica have influenced both tetraploid transcriptomes as suggested by inheritance patterns of gene expression, parental contributions to tetraploid transcriptomes, and homeolog expression bias.

\section{Conclusion}

The present comparative transcriptome analysis revealed that two independently originated Achillea allotetraploid species exhibited difference in gene expression, some of which was inevitably produced by randomly combined effects of hybridization and polyploidization, but some others must have occurred and maintained under natural selection and/or genetic drift during their tens of thousand years of evolution [30]. Particularly, the species-specific expressed genes enriched for GO terms pertaining to "defense response" suggested differential adaptation during their post-speciation evolution. On the other hand, they showed similar transcriptome changes in comparison to their diploid progenitors. This similarity may be expected when the combinations of genomes merged by different allopolyploidization events were similar [37]. More detailed studies are now required to determine the adaptive significance of differences in gene expression between these two allotetraploid species, which have been revealed by our analysis.

\section{Methods}

\section{Plant materials}

Plants used for this study were grown in laboratory incubators (16 h: $8 \mathrm{~h}$ light-dark cycles at $23^{\circ} \mathrm{C}$ ) for $3-4$ months from achenes collected from natural populations of the four Achillea species in China. Achenes of the allotetraploid A. alpina, were sampled from Arxan Mountain in the northeast $\left(\mathrm{N} 47^{\circ} 17^{\prime}\right.$, E $\left.120^{\circ} 27^{\prime} ; 860 \mathrm{~m}\right)$, where both diploid species also occur in sympatry. Achenes of the other tetraploid, A. wilsoniana in the southwest, were collected from Taibai Mountain (N 33⒌', E $107^{\circ} 17^{\prime}$; alt. $2094 \mathrm{~m}$ ) in Qinling mountain range, approximately where this allotetraploid was originated. Because populations of diploid species A. acuminata in NE China and in Qinling mountains are genetically differentiated [30], achenes of this species were collected from both Arxan Mt. and Taibai Mt.. In contrast, we collected achenes of diploid $A$. asiatica only from Arxan Mt. as populations of this species across E Asia are genetically similar [30].

Tissues analyzed were stem apex and the first fullyspread leaf beneath the stem apex. Samples of different tissues were separately snap frozen in liquid nitrogen and stored at $-80^{\circ} \mathrm{C}$. Three replicates of each tissue were obtained, with each replicate containing samples pooled from several plant individuals so that there was sufficient RNA for analysis.

\section{RNA extraction, cDNA library construction and RNA sequencing}

Total RNA was extracted using a RNeasy Plus Mini Kit (Qiagen, Hilden, Germany). RNA concentration and quantification were determined using the NanoDrop 2000 spectrophotometer (Thermo Scientific, USA). RNAsequencing libraries of each sample were constructed and sequenced on an Illumina Hiseq 2000 platform with 100 bp paired-end reads by the Biodynamic Optical Imaging Center (BIOPIC) of Peking University (Beijing, China). The sequencing data have been deposited with links to BioProject accession number PRJNA669168 in the NCBI BioProject database (https://www.ncbi.nlm.nih.gov/ bioproject/PRJNA669168).

\section{RNA-Seq de novo assembly and annotation}

The number and quality of raw reads from each library were evaluated with FastQC v. 0.11.2 (http://www. bioinformatics.babraham.ac.uk/projects/fastqc). Adapter sequences, low quality bases $(\mathrm{Q}<20)$ and unknown nucleotides (Ns) were trimmed using Trimmomatic v. 0.32 [55]. After trimming, both end of reads with length above 25 bases were kept for assembly. To minimize technical bias, all filtered clean reads from three biological replicates were used to conduct de novo transcriptome assembly by Trinity (v. r2013-02-25) [56]. Redundant transcripts were removed using CD-HIT (v. 4.6) [57] with $90 \%$ identity, and the longest transcript in each group was retained. Positively expressed genes were defined using an empirical cutoff value (FPKM $>1)$, and those with more than $200 \mathrm{bp}$ were chosen as reliable unigenes [58]. The recognition of ORFs (open reading frames) and lncRNA were conducted on unigenes by TransDecoder (v. 2.0.1) (http://transdecoder.github.io/) and $\mathrm{CNCI}$, separately, and unigenes with a complete or partial ORF were prepared for subsequent gene expression analysis [59].

Functions of unigenes were identified by searching against NCBI NR databases using locally installed BLASTX with an E-value cutoff of $1 \mathrm{e}-5$, and the best alignment results were assigned as annotations of unigenes. The same strategy was applied to searches in Uniprot and KEGG databases.

\section{Ortholog and homeolog identification}

All unigene sequences were aligned using BLASTN with cutoff E-value of 1e-10, with orthologous gene families identified by OrthoFinder (v. 0.4.0) [60, 61]. BLAST similarity searches were performed for pairwise 
comparisons between libraries. Orthologous genes were standardized by setting E-value $\leq 5 \mathrm{e}-100$, alignment length $\geq 200 \mathrm{bp}$, and identity $\geq 90 \%$.

Previous data showed that the two progenitors of the studied allotetraploids are genetically distinct, and the allotetraploid species have maintained their parental subgenomes relatively intact $[30,32,33]$. This made it easy to identify homeologous gene copies in the allotetraploid species using the single nucleotide polymorphisms (SNPs) between the two diploid species. Clean reads of diploid and allotetraploid species were mapped to the assembled unigenes of the two allotetraploids, and SNPs were identified by SAMtools (v0.1.17) [62]. Only SNPs that could tell the genomes of the parental species $A$. acuminata and $A$. asiatica apart were chosen, and clean reads in the allotetraploids exhibiting parental SNPs were parsed into homeolog-specific bins using custom perl scripts so that reads in the tetraploids were designated as of A. acuminata- or A. asiatica-type.

\section{Differential expression among species}

Species-specific expressed and species-coexpressed genes were identified using orthologous gene families as units. Only genes coexpressed in the allopolyploid and both of parental species were further analyzed for gene expression levels. The number of clean reads mapped onto each gene was counted by RSEM (v. 1.1.13) [63] and the expression level of an unigene was determined as the average of three biological replicates. The analysis for differential expression between an allopolyploid and each of its diploid progenitors was performed using edgeR (v. 2.2.5) in R software (v. 2.13) with the trimmed mean of M-values (TMM) to normalize read counts within and across libraries. Benjamini and Hochberg $(\mathrm{BH})$ methods were used to adjust $p$-values to account for significance of differentially expressed genes (DEGs) $[64,65]$. DEGs were identified by absolute value of $\log _{2}$ (fold change) $>1$ and FDR $<0.05$ using a negative binomial test. DEGs among the allotetraploid and its diploid progenitors were assigned to 12 categories modified from Rapp et al. (2009) [48] containing intermediate expression of the polyploids between that of the parents, expression-level dominance, transgressive expression, and conserved (equal in all species).

\section{Analyses of homeolog expression bias}

To calculate the expression levels of homeologs in the allotetraploid, read number mapped onto putative parental interspecific SNPs was counted and the average of those read number was calculated when more than one such SNP occurred in one fragment. To understand the homeolog-specific contributions to the allotetraploid gene expression, the analysis of differential expression was assessed between the two parental homeologs via a negative binomial test in edge $\mathrm{R}$ package with the criterion of absolute value of $\log _{2}$ (fold change) $>1, \mathrm{FDR}<0.05$ and $P$-value $<0.05$. To further quantify expression level differences, we defined the relative expression of homeologs (Rh) as: $\mathrm{Rh}=\log _{2}$ (acu-homeolog/asi-homeolog), where acu-homeolog or asi-homeolog is the expression level of the corresponding homeolog. This measurement can be computed for any homeolog pair with non-zero read counts (testable homeolog pairs); when $\mathrm{Rh}>0$, it indicates a higher expression level of the $A$. acuminata-homeolog than the $A$. asiatica-homeolog, and vice versa, when $\mathrm{Rh}<$ 0 , the $A$. asiatica-homeolog may be expressed higher.

To examine the homeolog expression bias, we further defined the relative expression level of orthologous pairs of genes in parental species (Rp) as: $\mathrm{Rp}=\log _{2}$ (A. acuminata/A. asiatica) and compared $\mathrm{Rh}$ with $\mathrm{Rp}$ using Fisher's exact tests with the criterion of absolute value of $\log _{2}$ (fold change) $>1$, FDR $<0.01$ and P-value $<0.05$. When $\mathrm{Rh}>\mathrm{Rp}$, it indicates expression bias toward diploid $A$. acuminata, and vice versa, when $\mathrm{Rh}<\mathrm{Rp}$, expression bias toward diploid $A$. asiatica.

\section{Validation of DEGs by reverse transcription real-time quantitative PCR (RT-qPCR)}

To confirm the differential gene expression presented by the RNA-Seq data, we performed reverse transcription Real-Time quantitative PCR (RT-qPCR) analysis on several randomly selected genes. Gene-specific primer pairs were designed by the Primer Premier 5.0. Tissue/organ samples were the same as for the RNA-Seq analysis. Three independent batches of RNA were isolated as biological replicates. The Fast Quant RT kit (with gDNase) (Tiangen Biotech, Beijing, China) was used for cDNA synthesis following the manufacturer's instructions. Then SYBR Premix Ex TaqTM (Tli RNaseH Plus) (Takara) was used for $\mathrm{GPCR}$ reactions. PCR reactions were performed on a 7500/7500 Fast Real-Time PCR System (Applied Biosystems) with the following program: $95^{\circ} \mathrm{C}$ for $5 \mathrm{~min}$, and then 40 PCR cycles at $95^{\circ} \mathrm{C}$ for $5 \mathrm{~s} ; 60^{\circ} \mathrm{C}$ for $34 \mathrm{~s}$. The glucose 6-phosphate dehydrogenase $(\mathrm{G} 6 \mathrm{PDH})$ and protein phosphatase 2A (PP2A) genes, which were confirmed having similar expression level among all the studied species by RNA-seq and qRT-PCR, were used as the internal reference genes. A relative quantitative method (delta-delta $\mathrm{Ct}$ ) was used to evaluate the expression level of candidate genes. Primers used in this study are listed in Table S3.

\section{Supplementary Information}

The online version contains supplementary material available at https://doi. org/10.1186/s12864-021-07566-6.

Additional file 1: Supplementary Fig. S1. Correlation analysis of transcriptome data from different samples. 
Additional file 2: Supplementary Fig. S2. Patterns of gene expression detected by RT-qPCR to verify the RNA-Seq data.

Additional file 3: Supplementary Table S1. Information of reads in the transcriptome data.

Additional file 4: Supplementary Table S2. Relative expression leve and expression bias of homeologs in the studied Achillea allotetraploid species.

Additional file 5: Supplementary Table S3. Primers used in RT-qPCR assays.

Additional file 6. Full information of enriched $\mathrm{GO}$ terms of genes specifically coexpressed by each allotetraploid species with each of its diploid progenitors as shown in Fig. 1. The top ten most enriched GO terms related to biological process (BP) of genes specifically coexpressed of each allotetraploid species with its sympatric diploid A. acuminata are shown in Fig. 2.

Additional file 7. Full information of enriched GO terms of (1) speciesspecifically expressed genes when the two allotetraploid species are compared, and (2) population-specifically expressed genes when two populaitons of the diploid parental $A$. acuminata are compared. The top ten most enriched $\mathrm{GO}$ terms related to biological process (BP) are shown in Fig. 2 .

\section{Acknowledgements}

We are grateful to Richard J. Abbott (University of St Andrews, UK) for constructive advices and comments for this manuscript.

\section{Authors' contributions}

DC designed and performed the experiments, interpreted the RNA-Seq data and wrote the draft of the manuscript. PCY analyzed the RNA-Seq data. YPG conceived the research, led the manuscript writing. All authors read and approved the final manuscript.

\section{Funding}

This work was supported financially by the National Natural Science Foundation of China (Grant Nos. 32070234 and 31570215), and the 111 project (B13008) of China.

\section{Availability of data and materials}

The data generated and analyzed by this study have been deposited with links to BioProject accession number PRJNA669168 in the NCBI BioProject database (https://www.ncbi.nlm.nih.gov/bioproject/PRJNA669168).

\section{Declarations}

\section{Ethics approval and consent to participate}

The plants under this study are not rare or endangered. The samples were collected in their wild populations in non-protected areas; no any legal authorization/license is required.

\section{Consent for publication}

Not applicable.

\section{Competing interests}

The authors declare that they have no competing interests.

\section{Author details}

${ }^{1}$ Key Laboratory of Biodiversity Science and Ecological Engineering of the Ministry of Education, and College of Life Sciences, Beijing Normal University, Beijing, China. ${ }^{2}$ Beijing Tangtang Tianxia Biotechnology Co., Ltd, Beijing, China.

Received: 9 November 2020 Accepted: 30 March 2021

Published online: 13 April 2021

\section{References}

1. Hegarty MJ, Barker GL, Wilson ID, Abbott RJ, Edwards KJ, Hiscock SJ. Transcriptome shock after interspecific hybridization in Senecio is ameliorated by genome duplication. Curr Biol. 2006;16(16):1652-9. https:// doi.org/10.1016/j.cub.2006.06.071.
2. Doyle JJ, Flagel LE, Paterson AH, Rapp RA, Soltis DE, Soltis PS, et al. Evolutionary genetics of genome merger and doubling in plants. Ann Rev Genet. 2008; 42(1):443-61. https://doi.org/10.1146/annurev.genet.42.110807.091524.

3. Buggs RJ, Zhang L, Miles N, Tate JA, Gao L, Wei W, et al. Transcriptomic shock generates evolutionary novelty in a newly formed natural allopolyploid plant. Curr Biol. 2011;21(7):551-6. https://doi.org/10.1016/j. cub.2011.02.016.

4. Springer NM, Li Q, Lisch DJPC. Creating order from chaos: Epigenome dynamics in plants with complex genomes. Plant Cell. 2016;2016:314-25.

5. Van de Peer $Y$, Mizrachi $E$, Marchal K. The evolutionary significance of polyploidy. Nat Rev Genet. 2017;18(7):411-24. https://doi.org/10.1038/ nrg.2017.26.

6. Wendel JF, Lisch D, Hu G, Mason AS. The long and short of doubling down: polyploidy, epigenetics, and the temporal dynamics of genome fractionation. Curr Opin Genet Dev. 2018;49:1-7. https://doi.org/10.1016/j. gde.2018.01.004

7. Anderson E, Stebbins GL. Hybridization as an evolutionary stimulus. Evolution. 1954;8(4):378-88. https://doi.org/10.1111/j.1558-5646.1954. tb01504.x.

8. Grant V. Plant Speciation. New York: Columbia University Press; 1981. https://doi.org/10.7312/gran92318.

9. Otto SP. The evolutionary consequences of polyploidy. Cell. 2007;131(3): 452-62. https://doi.org/10.1016/j.cell.2007.10.022.

10. Chen ZJ. Genetic and epigenetic mechanisms for gene expression and phenotypic variation in plant polyploids. Ann Rev Plant Biol. 2007;58(1):377406. https://doi.org/10.1146/annurev.arplant.58.032806.103835.

11. Jiao YN, Wickett NJ, Ayyampalayam S, Chanderbali AS, Landherr L, Ralph PE, et al. Ancestral polyploidy in seed plants and angiosperms. Nature. 2011; 473(7345):97-100. https://doi.org/10.1038/nature09916.

12. Abbott R, Albach D, Ansell S, Arntzen JW, Baird SJE, Bierne N, et al. Hybridization and speciation. J Evol Biol. 2013;26(2):229-46. https://doi.org/1 0.1111/j.1420-9101.2012.02599.x.

13. Soltis DE, Visger CJ, Soltis PS. The polyploidy revolution then ... and now: Stebbins revisited. Amer J Bot. 2014;101(7):1057-78. https://doi.org/10.3 732/ajb.1400178.

14. Kashkush K, Feldman M, Levy AA. Gene loss, silencing and activation in a newly synthesized wheat allotetraploid. Genetics. 2002;160(4):1651-9.

15. Wang J, Tian L, Lee H-S, Wei NE, Jiang H, Watson B, et al. Genomewide nonadditive gene regulation in Arabidopsis allotetraploids. Genetics. 2006; 172(1):507-17. https://doi.org/10.1534/genetics.105.047894.

16. Zhang D, Pan Q, Tan C, Zhu B, Ge X, Shao Y, et al. Genome-wide gene expressions respond differently to A-subgenome origins in Brassica napus synthetic hybrids and natural allotetraploid. Front Plant Sci. 2016;7:1508.

17. Wu J, Lin L, Xu M, Chen P, Liu D, Sun Q, et al. Homoeolog expression bias and expression level dominance in resynthesized allopolyploid Brassica napus. BMC Genomics. 2018;19:1-13.

18. Yoo M, Szadkowski E, Wendel J. Homoeolog expression bias and expression level dominance in allopolyploid cotton. Heredity. 2013;110(2):171-80. https://doi.org/10.1038/hdy.2012.94.

19. Mandáková T, Lysak MA. Post-polyploid diploidization and diversification through dysploid changes. Curr Opin Plant Biol. 2018;42:55-65. https://doi. org/10.1016/j.pbi.2018.03.001

20. Soltis DE, Soltis PS. Polyploidy: recurrent formation and genome evolution Trends Ecol Evol. 1999;14(9):348-52. https://doi.org/10.1016/S0169-534 7(99)01638-9.

21. Soltis D, Buggs R, Barbazuk W, Schnable P, Soltis P. On the origins of species: does evolution repeat itself in polyploid populations of independent origin? Cold Spring Harb Symp Quant Biol. 2009;74:215-23. https://doi.org/10.1101/sqb.2009.74.007.

22. Tate JA, Ni Z, Scheen AC, Koh J, Gilbert CA, Lefkowitz D, et al. Evolution and expression of homoeologous loci in Tragopogon miscellus (Asteraceae), a recent and reciprocally formed allopolyploid. Genetics. 2006;173(3):1599611. https://doi.org/10.1534/genetics.106.057646.

23. Buggs RJA, Doust AN, Tate JA, Koh J, Soltis K, Feltus FA, et al. Gene loss and silencing in Tragopogon miscellus (Asteraceae): comparison of natural and synthetic allotetraploids. Heredity. 2009;103(1):73-81. https://doi.org/10.103 8/hdy.2009.24

24. Tate JA, Joshi P, Soltis KA, Soltis PS, Soltis DE. On the road to diploidization? Homoeolog loss in independently formed populations of the allopolyploid Tragopogon miscellus (Asteraceae). BMC Plant Biol. 2009;9(1):80-9. https:// doi.org/10.1186/1471-2229-9-80. 
25. Koh J, Soltis PS, Soltis DE. Homoeolog loss and expression changes in natural populations of the recently and repeatedly formed allotetraploid Tragopogon mirus (Asteraceae). BMC Genomics. 2010;11(1):97-121. https:// doi.org/10.1186/1471-2164-11-97.

26. Buggs RJA, Chamala S, Wu W, Gao L, May GD, Schnable PS, et al. Characterization of duplicate gene evolution in the recent natural allopolyploid Tragopogon miscellus by next-generation sequencing and Sequenom iPLEX MassARRAY genotyping. Mole Ecol. 2010;19:132-46. https://doi.org/10.1111/j.1365-294X.2009.04469.x

27. Chester M, Gallagher JP, Symonds W, Cruz da Silva AV, Mavrodiev EV, Leitch $A R$, et al. Extensive chromosomal variation in a recently formed natural allopolyploid species, Tragopogon miscellus (Asteraceae). Proc Nat Acad Sci USA. 2012;109:1176-81.

28. Paun O, Bateman RM, Fay MF, Hedrén M, Civeyrel L, Chase MW. Stable epigenetic effects impact adaptation in allopolyploid orchids (Dactylorhiza: Orchidaceae). Mole Biol Evol. 2010;27(11):2465-73. https://doi.org/10.1093/ molbev/msq150.

29. Paun O, Bateman RM, Fay MF, Luna JA, Moat J, Hedrén M, et al. Altered gene expression and ecological divergence in sibling allopolyploids of Dactylorhiza (Orchidaceae). BMC Evol Biol. 2011;11(1):113. https://doi.org/1 0.1186/1471-2148-11-113

30. Wan JN, Guo YP, Rao GY. Unraveling independent origins of two tetraploid Achillea species by amplicon sequencing. J Syst Evol. 2020;58(6):913-24. https://doi.org/10.1111/jse.12544.

31. Shih C, Fu GX. Achillea L. In: Flora Reipublicae Popularis Sinica, vol. 76. Beijing: Science Press; 1983. p. 9-19.

32. Guo YP, Vogl C, van Loo M, Ehrendorfer F. Hybrid origin and differentiation of two tetraploid Achillea species in East Asia: molecular, morphological and ecogeographical evidence. Mole Ecol. 2006;15:133-44.

33. Guo YP, Tong XY, Wang LW, Vogl C. A population genetic model to infer allotetraploid speciation and long-term evolution applied to two yarrow species. New Phytol. 2013;199(2):609-21. https://doi.org/10.1111/nph.12262.

34. Grover C, Gallagher J, Szadkowski E, Yoo M, Flagel L, Wendel J. Homoeolog expression bias and expression level dominance in allopolyploids. New Phytol. 2012;196(4):966-71. https://doi.org/10.1111/j.1469-8137.2012.04365.x.

35. Buggs RJ, Wendel JF, Doyle JJ, Soltis DE, Soltis PS, Coate JE. The legacy of diploid progenitors in allopolyploid gene expression patterns. Philos Trans R Soc B Biol Sci. 2014;369(1648):20130354. https://doi.org/10.1098/ rstb.2013.0354.

36. Yoo MJ, Liu X, Pires JC, Soltis PS, Soltis DE. Nonadditive gene expression in polyploids. Ann Rev Genet. 2014;48(1):485-517. https://doi.org/10.1146/a nnurev-genet-120213-092159.

37. Bottani S, Zabet NR, Wendel JF, Veitia RA. Gene expression dominance in allopolyploids: hypotheses and models. Trends Plant Sci. 2018;23(5):393-402. https://doi.org/10.1016/j.tplants.2018.01.002.

38. Guo YP, Ehrendorfer F, Samuel R. Phylogeny and systematics of Achillea (Asteraceae-anthemideae) inferred from the nrITS and plastid trnL-F DNA sequences. Taxon. 2004;53(3):657-72. https://doi.org/10.2307/4135441.

39. Ramsey J. Polyploidy and ecological adaptation in wild yarrow. Proc Natl Acad Sci U S A. 2011;108(17):7096-101. https://doi.org/10.1073/pna s.1016631108

40. Uller T. Developmental plasticity and the evolution of parental effects. Trends Ecol Evol. 2008;23(8):432-8. https://doi.org/10.1016/j.tree.2008.04.005.

41. Donohue K. Completing the cycle: maternal effects as the missing link in plant life histories. Philos Trans R Soc Lond Ser B Biol Sci. 2009;364(1520): 1059-74. https://doi.org/10.1098/rstb.2008.0291.

42. Videvall E, Sletvold N, Hagenblad J, Ågren J, Hansson B. Strong maternal effects on gene expression in Arabidopsis lyrata hybrids. Mol Biol Evol. 2016; 33(4):984-94. https://doi.org/10.1093/molbev/msv342.

43. Gong L, Salmon A, Yoo M-J, Grupp KK, Wang Z, Paterson AH, et al. The cytonuclear dimension of allopolyploid evolution: an example from cotton using rubisco. Mol Biol Evol. 2012;29(10):3023-36. https://doi.org/10.1093/ molbev/mss110.

44. Shi XL, Ng DWK, Zhang CQ, Comai L, Ye WX, Chen ZJ. Cis-and transregulatory divergence between progenitor species determines geneexpression novelty in Arabidopsis allopolyploids. Nat Commun. 2012;3(1):950. https://doi.org/10.1038/ncomms1954.

45. Combes MC, Hueber Y, Dereeper A, Rialle S, Herrera JC, Lashermes P. Regulatory divergence between parental alleles determines gene expression patterns in hybrids. Genome Biol Evol. 2015;7(4):1110-21. https://doi.org/1 0.1093/gbe/evv057.
46. Parisod C, Alix K, Just J, Petit M, Sarilar V, Mhiri C, et al. Impact of transposable elements on the organization and function of allopolyploid genomes. New Phytol. 2010;186(1):37-45. https://doi.org/10.1111/j.1469-813 7.2009.03096.x.

47. Vicient CM, Casacuberta JM. Impact of transposable elements on polyploid plant genomes. Ann Bot. 2017;120(2):195-207. https://doi.org/10.1093/aob/mcx078.

48. Rapp RA, Udall JA, Wendel JF. Genomic expression dominance in allopolyploids. BMC Biol. 2009;7(1):18. https://doi.org/10.1186/17417007-7-18.

49. Chelaifa H, Monnier A, Ainouche M. Transcriptomic changes following recent natural hybridization and allopolyploidy in the salt marsh species Spartina $\times$ townsendii and Spartina anglica (Poaceae). New Phytol. 2010; 186(1):161-74. https://doi.org/10.1111/j.1469-8137.2010.03179.x

50. Qi B, Huang W, Zhu B, Zhong XF, Guo JH, Zhao N, et al. Global transgenerational gene expression dynamics in two newly synthesized allohexaploid wheat (Triticum aestivum) lines. BMC Biol. 2012;10(1):3. https:// doi.org/10.1186/1741-7007-10-3.

51. Soltis DE, Buggs RJ, Barbazuk WB, Chamala S, Chester M, Gallagher JP, et al. The early stages of polyploidy: rapid and repeated evolution in Tragopogon. In: Soltis PS, Soltis DE, editors. Polyploidy and genome evolution. Berlin: Springer; 2012. p. 271-92. https://doi.org/10.1007/ 978-3-642-31442-1_14.

52. Clausen J, Keck DD, Hiesey WM. Experimental studies on the nature of species. III. Environmental Responses of Climatic Races of Achillea. Washington: Carnegie Institution of Washington Publication; 1948. No. 581

53. Ramsey J, Robertson A, Husband B, Conti E. Rapid adaptive divergence in New World Achillea, an autopolyploid complex of ecological races. Evolution. 2008; 62(3):639-53. https:/doi.org/10.1111/j.1558-5646.2007.00264.x.

54. Chao DY, Dilkes B, Luo HB, Douglas A, Yakubova E, Lahner B, et al. Polyploids exhibit higher potassium uptake and salinity tolerance in Arabidopsis. Science. 2013;341(6146):658-9. https://doi.org/10.1126/science.1240561.

55. Cox MP, Peterson DA, Biggs PJ. SolexaQA: at-a-glance quality assessment of Illumina second-generation sequencing data. BMC Bioinformatics. 2010; 11(1):485. https://doi.org/10.1186/1471-2105-11-485.

56. Grabherr MG, Haas BJ, Yassour M, Levin JZ, Thompson DA, Amit I, et al. Full-length transcriptome assembly from RNA-Seq data without a reference genome. Nat Biotechnol. 2011;29(7):644-52. https://doi.org/1 0.1038/nbt.1883.

57. Li WZ, Godzik A. Cd-hit: a fast program for clustering and comparing large sets of protein or nucleotide sequences. Bioinformatics. 2006;22(13):1658-9. https://doi.org/10.1093/bioinformatics/btl158.

58. Trapnell C, Williams BA, Pertea G, Mortazavi A, Kwan G, van Baren MJ, et al. Transcript assembly and abundance estimation from RNA-Seq reveals thousands of new transcripts and switching among isoforms. Nat Biotechnol. 2010;28(5):511-5. https://doi.org/10.1038/nbt.1621.

59. Liang S, Luo HT, Bu DC, Zhao GG, Yu KT, Zhang CH, et al. Utilizing sequence intrinsic composition to classify protein-coding and long non-coding transcripts. Nucleic Acids Res. 2013;41:e166.

60. Shiryev SA, Papadopoulos JS, Schaeffer AA, Agarwala R. Improved BLAST searches using longer words for protein seeding. Bioinformatics. 2007; 23(21):2949-51. https://doi.org/10.1093/bioinformatics/btm479.

61. Emms DM, Kelly S. OrthoFinder: solving fundamental biases in whole genome comparisons dramatically improves orthogroup inference accuracy. Genome Biol. 2015;16(1):157. https://doi.org/10.1186/s13059-015-0721-2.

62. Li H, Handsaker B, Wysoker A, Fennell T, Ruan J, Homer N, et al. 1000 genome project data processing subgroup. The sequence alignment/map format and SAMtools. Bioinformatics. 2009;25(16):2078-9. https://doi.org/1 0.1093/bioinformatics/btp352.

63. Li B, Dewey CN. RSEM: accurate transcript quantification from RNA-Seq data with or without a reference genome. BMC Bioinformatics. 2011;12(1):323. https://doi.org/10.1186/1471-2105-12-323.

64. Benjamini $Y$, Hochberg Y. Controlling the false discovery rate: a practical and powerful approach to multiple testing. J R Stat Soc: Series B. 1995;57: 289-300.

65. Robinson MD, Oshlack A. A scaling normalization method for differential expression analysis of RNA-Seq data. Genome Biol. 2010;11(3):R25. https:// doi.org/10.1186/gb-2010-11-3-r25.

\section{Publisher's Note}

Springer Nature remains neutral with regard to jurisdictional claims in published maps and institutional affiliations. 